\title{
Numerical approach to the semiclassical method of radiation emission for arbitrary electron spin and photon polarization
}

\author{
T. N. Wistisen $\odot$ and A. Di Piazza $\odot$ \\ Max-Planck-Institut für Kernphysik, Saupfercheckweg 1, D-69117 Heidelberg, Germany
}

(Received 1 October 2019; published 2 December 2019)

\begin{abstract}
We show how the semiclassical formulas for radiation emission of Baier, Katkov and Strakhovenko for arbitrary initial and final spins of the electron and arbitrary polarization of the emitted photon can be rewritten in a form which numerically converges quickly. We directly compare the method in the case of a background plane wave with the result obtained by using the Volkov state solution of the Dirac equation, and confirm that we obtain the same result. We then investigate the interaction of a circularly polarized short laser pulse scattering with $\mathrm{GeV}$ electrons and see that the finite duration of the pulse leads to a lower transfer of circular polarization than that predicted by the known formulas in the monochromatic case. We also see how the transfer of circular polarization from the laser beam to the gamma ray beam is gradually deteriorated as the laser intensity increases, entering the nonlinear regime. However, this is shown to be recovered if the scattered photon beam is collimated to only allow for passage of photons emitted with angles smaller than $1 / \gamma$ with respect to the initial electron direction, where $\gamma$ is the approximately constant Lorentz factor of the electron. The obtained formulas also allow us to answer questions regarding radiative polarization of the emitting particles. In this respect we briefly discuss an application of the present approach to the case of a bent crystal and high-energy positrons.
\end{abstract}

DOI: 10.1103/PhysRevD.100.116001

\section{INTRODUCTION}

In view of recent and upcoming experiments involving radiation emission from relativistic electrons [1-5] and of high-intensity laser facilities under construction like the Extreme Light Infrastructure (ELI), it is fitting to investigate efficient approaches to calculating the high-energy radiation emitted when ultrarelativistic electrons collide with such an intense laser field with as much generality as possible. The semiclassical formalism of Baier, Katkov and Strakhovenko allows for the approximate determination of the spectrum of emitted photons from an ultrarelativistic electron in a virtually arbitrary external electromagnetic field [6]. For numerical applications the formulation with a single time integration, as found in $[7,8]$ for the spin and polarization averaged result, is most useful. In this paper we show how the basic result of the semiclassical method with explicit electron spin and photon polarization can also be treated numerically in a similar fashion. We use the obtained formulas in the case of a background plane wave, as the Dirac equation then can be solved analytically [9], to do a direct comparison with the spectrum obtained using

Published by the American Physical Society under the terms of the Creative Commons Attribution 4.0 International license. Further distribution of this work must maintain attribution to the author(s) and the published article's title, journal citation, and DOI. Funded by SCOAP ${ }^{3}$. the exact solution of the Dirac equation (Volkov states) [9-11]. This is the usual approach for such processes [12-27]. We consider the case of a short circularly polarized laser pulse and find agreement, as expected. The advantage of the presented approach is the possibility of calculating the radiation emission under general circumstances, i.e., also for very complicated field configurations as one only needs the classical trajectory in the external field, which can easily be found numerically for a given field. The presented formulas allow us to find the polarization properties of the radiation depending on the spin of the initial and final electrons, which also allows us to determine if the electrons become polarized. The latter would occur if the spin-flip radiation has a different yield for each of the possible initial spin states (see e.g., [28]), i.e., a generalization of the Sokolov-Ternov effect [29] to fields other than that of a permanent magnetic field [30,31]. We briefly demonstrate this in the case of positrons channeling in a bent germanium crystal, where one has two kinds of motion superimposed-the oscillatory channeling motion between the bent planes, which in the unbent case would not lead to polarization, along with the motion along the bending arc, which leads to transverse polarization of the positrons. When the crystal is strongly bent, i.e., close to the so-called Tsyganov radius [32,33], the polarization as in a magnetic field is obtained, while smaller bending radii lead to smaller degrees of polarization, which the presented method allows us to predict. 
Below, $e$ indicates the positron charge, and units are used, such that the fine-structure constant $\alpha$ is given by $e^{2}$, whereas the relativistic metric +--- is employed. We use Feynman notation to write $\not h=a_{\mu} \gamma^{\mu}$, where $a^{\mu}$ is a generic 4-vector.

\section{SEMICLASSICAL APPROACH}

Below, we study the emission by an electron of a single photon in a given background electromagnetic field. The basic result of the semiclassical method of Baier et al. in its most general form for the single-photon radiation probability is expressed as [6]

$$
d P=\frac{\alpha \omega}{(2 \pi)^{2}}\left|\int_{-\infty}^{\infty} R(t) e^{i k^{\prime} x} d t\right|^{2} d \Omega d \omega,
$$

where $x^{\mu}=\{t, \boldsymbol{x}(t)\}$ is the electron 4-position as obtained by the Lorentz force equation in the external field, $k^{\prime \mu}=\omega^{\prime}\{1, \boldsymbol{n}\}, \omega^{\prime}=\frac{\varepsilon}{\varepsilon^{\prime}} \omega, \omega$ is the energy of the emitted photon, $\varepsilon^{\prime}=\varepsilon-\omega, \varepsilon$ the initial electron energy, $\boldsymbol{n}$ the direction of emission, and

$$
R(t)=\phi_{f}^{\dagger}[A(t)+i \boldsymbol{\sigma} \cdot \boldsymbol{B}(t)] \phi_{i} .
$$

Here, $\phi_{i}$ and $\phi_{f}$ are the spinors of the initial and final electron states (characterized by the electron 4-momentum and the electron spin in its asymptotic rest frame), $\sigma$ denotes the vector of the Pauli spin matrices, and

$$
\begin{gathered}
A(t)=C \boldsymbol{\epsilon}^{*} \cdot \boldsymbol{v}(t), \\
\boldsymbol{B}(t)=\boldsymbol{\epsilon}^{*} \times\left[D_{1} \boldsymbol{v}(t)+D_{2} \boldsymbol{n}\right],
\end{gathered}
$$

with $\boldsymbol{\epsilon}$ being the polarization vector of the emitted photon, $\boldsymbol{v}(t)=d \boldsymbol{x}(t) / d t$ being the electron velocity, and the constants being given by

$$
\begin{gathered}
C=\frac{\varepsilon}{2 \sqrt{\varepsilon \varepsilon^{\prime}}}\left[\sqrt{\frac{\varepsilon^{\prime}+m}{\varepsilon+m}}+\sqrt{\frac{\varepsilon+m}{\varepsilon^{\prime}+m}}\right], \\
D_{1}=\frac{\varepsilon}{2 \sqrt{\varepsilon \varepsilon^{\prime}}}\left[\sqrt{\frac{\varepsilon^{\prime}+m}{\varepsilon+m}}-\sqrt{\frac{\varepsilon+m}{\varepsilon^{\prime}+m}}\right], \\
D_{2}=\frac{\omega}{2 \sqrt{\varepsilon \varepsilon^{\prime}}} \sqrt{\frac{\varepsilon+m}{\varepsilon^{\prime}+m}}
\end{gathered}
$$

where $m$ is the electron mass. To evaluate the quantity in Eq. (1) we need to carry out the two time integrals $\int \boldsymbol{v}(t) e^{i k^{\prime} x} d t$ and $\int e^{i k^{\prime} x} d t$. However, a direct computation of these integrals converges slowly, and integrations beyond times when the acceleration is different from zero must be included, as explained classically in [34]. From the relations shown in [8], which are already used there in the case without polarization and spin averaging, it is quite easy to relate these quantities to the quantities whose integrands are proportional to the acceleration. By doing this, we have that

$$
\begin{gathered}
\int_{-\infty}^{\infty} \boldsymbol{v}(t) e^{i k^{\prime} x} d t=\frac{i}{\omega^{\prime}}(\boldsymbol{n} J-\boldsymbol{I}), \\
\int_{-\infty}^{\infty} e^{i k^{\prime} x} d t=\frac{i}{\omega^{\prime}} J,
\end{gathered}
$$

where

$$
\begin{gathered}
\boldsymbol{I}=\int_{-\infty}^{\infty} \frac{\boldsymbol{n} \times[(\boldsymbol{n}-\boldsymbol{v}) \times \dot{\boldsymbol{v}}]}{(1-\boldsymbol{n} \cdot \boldsymbol{v})^{2}} e^{i k^{\prime} x} d t, \\
J=\int_{-\infty}^{\infty} \frac{\boldsymbol{n} \cdot \dot{\boldsymbol{v}}}{(1-\boldsymbol{n} \cdot \boldsymbol{v})^{2}} e^{i k^{\prime} x} d t .
\end{gathered}
$$

In [8] it is shown in detail how to calculate the electron trajectory and the quantities $I$ and $J$ numerically. In particular, it is appropriate to analytically carry out the cancellations between large terms, as in e.g., $1-\boldsymbol{n} \cdot \boldsymbol{v}$ because $\boldsymbol{n} \cdot \boldsymbol{v}$ is close to 1 for ultrarelativistic particles, as the radiation is emitted in a narrow cone around the direction of the velocity. Finally, we may write

$$
\begin{aligned}
\int_{-\infty}^{\infty} R(t) e^{i k^{\prime} x} d t= & -\frac{i}{\omega^{\prime}} \boldsymbol{\phi}_{f}^{\dagger}\left[\boldsymbol{C} \boldsymbol{\epsilon}^{*} \cdot \boldsymbol{I}\right. \\
& \left.+i \boldsymbol{\sigma} \cdot\left(\boldsymbol{\epsilon}^{*} \times\left[\boldsymbol{I} D_{1}-\left(D_{1}+D_{2}\right) \boldsymbol{n} J\right]\right)\right] \phi_{i},
\end{aligned}
$$

and therefore we obtain the emission probability as

$$
\begin{aligned}
\frac{d P}{d \Omega d \omega}= & \frac{\alpha}{(2 \pi)^{2}} \frac{\omega}{\omega^{\prime 2}} \times \mid \phi_{f}^{\dagger}\left[\boldsymbol{C \boldsymbol { \epsilon } ^ { * }} \cdot \boldsymbol{I}\right. \\
& \left.+i \boldsymbol{\sigma} \cdot\left(\boldsymbol{\epsilon}^{*} \times\left[\boldsymbol{I} D_{1}-\left(D_{1}+D_{2}\right) \boldsymbol{n} J\right]\right)\right]\left.\phi_{i}\right|^{2} .
\end{aligned}
$$

As this is a semiclassical approach there are limitations to its validity; however, these are difficult to find in practice. We discussed this in [35], but to briefly summarize, for the method to be valid, one should have ultrarelativistic particles, such that the angle of emission $\theta$ around the instantaneous velocity may be assumed to be small, $\theta \ll 1$. Furthermore, the motion should be meaningfully described by the classical trajectory; i.e., the principal quantum number connected with the motion should be large. Therefore, the approach works for relativistic particles in magnetic fields of laboratory field strengths but cannot describe the radiation from e.g., electrons closely bound in hydrogen. In [36] we showed how one could see slight differences in the semiclassical result and the fully quantum result for some cases in crystal channeling. 


\section{VOLKOV-STATE APPROACH}

If the background field is a plane wave, i.e., if the 4-vector potential $A^{\mu}(\varphi)$ only depends on the phase $\varphi=k_{0} x$, where $k_{0}=\left(\omega_{0}, \boldsymbol{k}_{0}\right)$ is the 4-momentum associated with the photons of the plane wave and $\omega_{0}=\left|\mathbf{k}_{0}\right|$, the corresponding Dirac equation

$$
(i \not \partial+e \not A-m) \psi=0
$$

can be solved analytically [9]. Below we assume that the plane wave propagates along the negative $z$ direction, and we choose the 4-vector potential $A^{\mu}(\varphi)$ in the Lorenz gauge where $A^{0}(\varphi)=A^{3}(\varphi)=0$. The positiveenergy solution reads

$$
\psi(x)=\frac{1}{\sqrt{2 \varepsilon}}\left(1-\frac{e \not k_{0} \not}{2 k_{0} p}\right) u e^{i S},
$$

where $p$ is the asymptotic 4-momentum of the electron (we have set the quantization volume equal to 1),

$$
S=-p x+\frac{e}{k_{0} p} \int^{\varphi} d \varphi^{\prime}\left[p A\left(\varphi^{\prime}\right)+\frac{e}{2} A^{2}\left(\varphi^{\prime}\right)\right]
$$

is the classical action of the electron in the plane wave, and $u$ is a short notation for the constant vacuum bispinor (which is characterized by the electron spin in the corresponding electron rest frame and by the electron 4-momentum $p$ ). The leading-order matrix element for single-photon emission is given by

$$
S_{f i}=i e \sqrt{\frac{4 \pi}{2 \omega}} \int d^{4} x \bar{\psi}_{f}(x) \phi^{*} e^{i k x} \psi_{i}(x),
$$

where $\psi_{i / f}(x)$ indicates the Volkov state corresponding to the initial/final electron state, and the differential probability of emission is then

$$
d P=\left|S_{f i}\right|^{2} \frac{d^{3} p_{f}}{(2 \pi)^{3}} \frac{d^{3} k}{(2 \pi)^{3}} .
$$

In the gauge we are working with, the 4-potential can be written as

$$
A^{\mu}(\varphi)=\sum_{j=1}^{2} a_{j}^{\mu} f_{j}(\varphi),
$$

where $a_{j}^{\mu}$ are two 4-vectors such that $a_{j} k_{0}=0$ and $a_{1} a_{2}=0$ and where $f_{j}(\varphi)$ are two arbitrary (physically wellbehaved) functions. By setting the arbitrary phase in the indefinite integrals in the phase of Volkov states to zero, we introduce the quantities

$$
\begin{aligned}
& F_{j}(\varphi)=\int_{0}^{\varphi} f_{j}\left(\varphi^{\prime}\right) d \varphi^{\prime}, \\
& G_{j}(\varphi)=\int_{0}^{\varphi} f_{j}^{2}\left(\varphi^{\prime}\right) d \varphi^{\prime} .
\end{aligned}
$$

Then, by inserting the expressions of Eqs. (15) and (19) into Eq. (17), we obtain that

$$
\begin{aligned}
S_{f i}= & i e \sqrt{\frac{4 \pi}{2 \omega}} \frac{1}{\sqrt{4 \varepsilon_{f} \varepsilon_{i}}} \int d^{4} x \\
& \times \bar{u}_{f}\left(\phi^{*}+\sum_{j=1}^{2}\left[B_{j} f_{j}(\varphi)+C_{j} f_{j}^{2}(\varphi)\right]\right) u_{i} \\
& \times e^{-i\left(p_{i}-p_{f}-k\right) x} e^{i\left(\sum_{j=1}^{2}\left[\alpha_{j} F_{j}(\varphi)+\beta_{j} G_{j}(\varphi)\right]\right)},
\end{aligned}
$$

where we have defined

$$
\begin{gathered}
\alpha_{j}=e\left[\frac{p_{i} a_{j}}{k_{0} p_{i}}-\frac{p_{f} a_{j}}{k_{0} p_{f}}\right], \\
\beta_{j}=\frac{e^{2} a_{j}^{2}}{2}\left[\frac{1}{k_{0} p_{i}}-\frac{1}{k_{0} p_{f}}\right],
\end{gathered}
$$

and

$$
\begin{aligned}
B_{j} & =-\left[\frac{e \not \phi_{j} \not k_{0}}{2 k_{0} p_{f}} \phi^{*}+\phi^{*} \frac{e \not k_{0} \not \not_{j}}{2 k_{0} p_{i}}\right], \\
C_{j} & =\frac{e \not \not_{j} \not_{0}}{2 k_{0} p_{f}} \phi^{*} \frac{e \not k_{0} \phi_{j}}{2 k_{0} p_{i}} \\
& =-\frac{e^{2} a_{j}^{2}}{2\left(k_{0} p_{f}\right)\left(k_{0} p_{i}\right)}\left(\epsilon^{*} k_{0}\right) \not k_{0},
\end{aligned}
$$

with $j=1,2$ [we have set $\epsilon^{\mu}=(0, \boldsymbol{\epsilon})$ ]. Now, we can write the functions in Eq. (22) as a Fourier transform

$f_{j}^{n}(\varphi) e^{i\left(\sum_{j=1}^{2}\left[\alpha_{j} F_{j}(\varphi)+\beta_{j} G_{j}(\varphi)\right]\right)}=\int_{-\infty}^{\infty} A_{n, j}(s, \alpha, \beta) e^{-i s \varphi} d s$,

where

$$
A_{n, j}(s, \alpha, \beta)=\frac{1}{2 \pi} \int_{-\infty}^{\infty} d \varphi f_{j}^{n}(\varphi) e^{i\left(s \varphi+\sum_{j=1}^{2}\left[\alpha_{j} F_{j}(\varphi)+\beta_{j} G_{j}(\varphi)\right]\right)},
$$

defined for $n=0,1,2$. When $n=0$, the $j$ subscript is superfluous, and we therefore denote this function as $A_{0}(s, \alpha, \beta)$. This function is however problematic as it diverges, but it can be regularized by using the identity (see also $[14,16,17])$ 


$$
0=\int_{-\infty}^{\infty} e^{i h(\varphi)} h^{\prime}(\varphi) d \varphi
$$

where

$$
h(\varphi)=s \varphi+\sum_{j=1}^{2}\left[\alpha_{j} F_{j}(\varphi)+\beta_{j} G_{j}(\varphi)\right] .
$$

In this way, we obtain

$$
\begin{aligned}
A_{0}(s, \alpha, \beta) & =\frac{1}{2 \pi} \int_{-\infty}^{\infty} e^{i\left(s \varphi+\sum_{j=1}^{2}\left[\alpha_{j} F_{j}(\varphi)+\beta_{j} G_{j}(\varphi)\right]\right)} d \varphi \\
& =-\frac{1}{s} \sum_{j=1}^{2}\left[\alpha_{j} A_{1, j}+\beta_{j} A_{2, j}\right] .
\end{aligned}
$$

By replacing these expressions in Eq. (22), and carrying out the integration over $d^{4} x$, we can write the amplitude in the form

$$
\begin{aligned}
S_{f i}= & i e \sqrt{\frac{4 \pi}{2 \omega}} \frac{1}{\sqrt{4 \varepsilon_{f} \varepsilon_{i}}} \int d s(2 \pi)^{4} \delta^{4}\left(p_{i}-p_{f}-k+s k_{0}\right) \\
& \times \bar{u}_{f}\left(\phi^{*} A_{0}+\sum_{j=1}^{2}\left[B_{j} A_{1, j}+C_{j} A_{2, j}\right]\right) u_{i}
\end{aligned}
$$

Now we can use the energy delta function to fix $s$ such that

$$
s_{0}=\frac{\varepsilon_{f}+\omega-\varepsilon_{i}}{\omega_{0}},
$$

and the delta function can be transformed as $\delta\left(\varepsilon_{i}-\varepsilon_{f}-\omega+s \omega_{0}\right)=\frac{1}{\omega_{0}} \delta\left(s-s_{0}\right):$

$$
\begin{aligned}
S_{f i}= & i e \sqrt{\frac{4 \pi}{2 \omega}} \frac{1}{\sqrt{4 \varepsilon_{f} \varepsilon_{i}}}(2 \pi)^{4} \frac{1}{\omega_{0}} \delta^{3}\left(\boldsymbol{p}_{i}-\boldsymbol{p}_{f}-\boldsymbol{k}+s_{0} \boldsymbol{k}_{0}\right) \\
& \times \bar{u}_{f}\left(\phi^{*} A_{0}+\sum_{j=1}^{2}\left[B_{j} A_{1, j}+C_{j} A_{2, j}\right]\right) u_{i}
\end{aligned}
$$

At this point we would then take the norm-square to obtain the transition probability; however, we are then faced with the problem of how to take the square of the delta-function, which has the complication that $s_{0}$ is a function of the momenta. The correct way to do this is to consider instead the more realistic case of an initial wave packet $\Psi_{i}(x)=$ $\int \psi_{\boldsymbol{p}_{i}}(x) c\left(\boldsymbol{p}_{i}\right) d^{3} \boldsymbol{p}_{i}$, where $\psi_{\boldsymbol{p}_{i}}(x)$ is the Volkov solution with momentum $\boldsymbol{p}_{i}$ and unindicated fixed spin quantum number. To preserve normalization we must have that $\int\left|c\left(\boldsymbol{p}_{i}\right)\right|^{2} d^{3} \boldsymbol{p}_{i}=1 /(2 \pi)^{3}$. Then the momentum deltafunction can be transformed as

$$
\delta^{3}\left(\boldsymbol{p}_{i}-\boldsymbol{p}_{f}-\boldsymbol{k}+s_{0}\left(\boldsymbol{p}_{i}\right) \boldsymbol{k}_{0}\right)=\frac{1}{\left|\mathcal{J}_{i}\right|} \delta^{3}\left(\boldsymbol{p}_{i}-\boldsymbol{p}_{i, \mathrm{sol}}\right),
$$

where $\mathcal{J}_{i}=\partial \boldsymbol{g} / \partial \boldsymbol{p}_{i}=I-\frac{\boldsymbol{k}_{0} \boldsymbol{p}_{i}^{T}}{\omega_{0} \varepsilon_{i}}$ is the Jacobian matrix, $I$ denotes the $3 \times 3$ identity matrix, and $\boldsymbol{g}\left(\boldsymbol{p}_{i}\right)=\boldsymbol{p}_{i}-\boldsymbol{p}_{f}-$ $\boldsymbol{k}+s_{0}\left(\boldsymbol{p}_{i}\right) \boldsymbol{k}_{0}, \boldsymbol{g}\left(\boldsymbol{p}_{i, \mathrm{sol}}\right)=\mathbf{0}\left(\boldsymbol{k}_{0} \boldsymbol{p}_{i}^{T}\right.$ indicates the dyadic product between the vectors $\boldsymbol{k}_{0}$ and $\boldsymbol{p}_{i}$ ); thus, using Sylvester's determinant theorem we obtain

$$
\left|\mathcal{J}_{i}\right|=\operatorname{det}\left(I-\frac{\boldsymbol{k}_{0} \boldsymbol{p}_{i}^{T}}{\omega_{0} \varepsilon_{i}}\right)=1-\frac{\boldsymbol{k}_{0} \cdot \boldsymbol{p}_{i}}{\omega_{0} \varepsilon_{i}}=\frac{k_{0} p_{i}}{\omega_{0} \varepsilon_{i}} .
$$

Therefore, we finally write the transition amplitude in the form

$$
\begin{aligned}
S_{f i}= & i e \sqrt{\frac{4 \pi}{2 \omega}} \frac{1}{\sqrt{4 \varepsilon_{f} \varepsilon_{i}}}(2 \pi)^{4} \frac{\varepsilon_{i}}{k_{0} p_{i}} \\
& \times c\left(\boldsymbol{p}_{f}+\boldsymbol{k}-s_{0}\left(\boldsymbol{p}_{i, \mathrm{sol}}, \boldsymbol{p}_{f}\right) \boldsymbol{k}_{0}\right) \\
& \times \bar{u}_{f}\left(\phi^{*} A_{0}+\sum_{j=1}^{2}\left[B_{j} A_{1, j}+C_{j} A_{2, j}\right]\right) u_{i} .
\end{aligned}
$$

Now, in order to find the probability using Eq. (18) we take the norm-square of the above amplitude and, having in mind the case of a narrow wave packet [27], replace $\left|c\left(\boldsymbol{p}_{i}\right)\right|^{2}=\delta^{3}\left(\boldsymbol{p}_{i}-\boldsymbol{p}_{i, 0}\right) /(2 \pi)^{3}$. Analogously as above, we now have a delta-function which we can evaluate by integration over $d^{3} \boldsymbol{p}_{f}$, and the transformation of the delta-function yields a factor of $\frac{\omega_{0} \varepsilon_{f}}{k_{0} p_{f}}$. Finally, we then obtain the differential emission probability

$$
\begin{aligned}
d P= & \left|\bar{u}_{f}\left(\phi^{*} A_{0}+\sum_{j=1}^{2}\left[B_{j} A_{1, j}+C_{j} A_{2, j}\right]\right) u_{i}\right|^{2} \\
& \times \frac{e^{2}}{4} \frac{1}{\left(k_{0} p_{i}\right)\left(k_{0} p_{f}\right)} \omega d \omega d \Omega,
\end{aligned}
$$

which can now be evaluated numerically. The bispinors in this expression are chosen as [37]

$$
u=\sqrt{\varepsilon+m}\left(\begin{array}{c}
\phi \\
\frac{\sigma \cdot p}{\varepsilon+m} \phi
\end{array}\right)
$$

where $\phi$ are spinors to be chosen as an orthonormal basis of eigenstates of $\boldsymbol{\sigma} \cdot \boldsymbol{s}$, with $\boldsymbol{s}$ being the direction of the otherwise arbitrary spin quantization axis in the rest frame of the electron.

\section{DISCUSSION OF RESULTS}

The above derivations were carried out without introducing a particular plane-wave pulse. We now consider a 


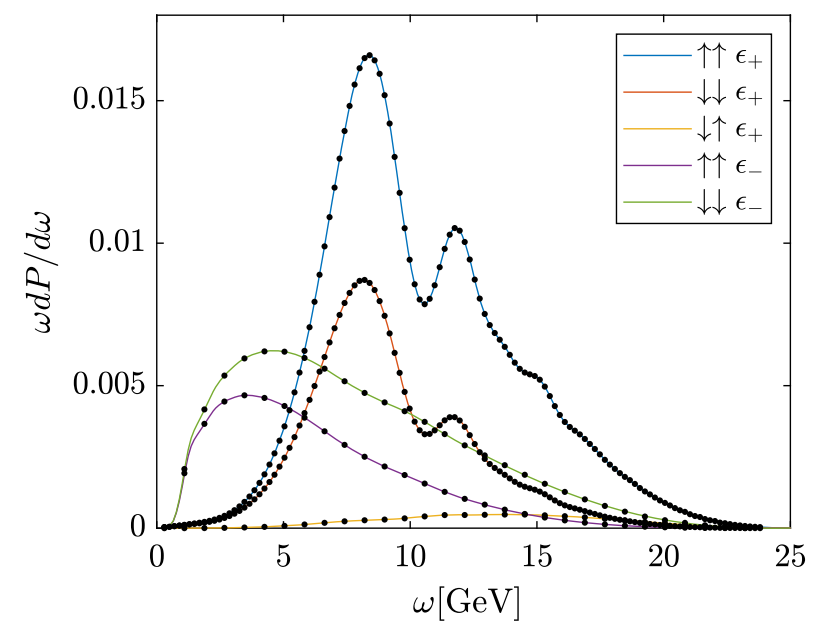

FIG. 1. The intensity spectrum $\omega d P / d \omega$ according to the semiclassical approach is shown as solid lines corresponding to the different possibilities of initial and final spins and photon polarizations. The first arrow denotes the initial electron spin, the second arrow the final electron spin, and the last symbol denotes the helicity state of the emitted radiation according to Eq. (44). The black dotted curves on top of the solid curves indicate the same quantities but calculated using the Volkov-states approach. We have not plotted the curve corresponding to $\uparrow \downarrow \boldsymbol{\epsilon}_{+}$and $\downarrow \uparrow \boldsymbol{\epsilon}_{-}$ as the corresponding yields are much smaller than the others and the curves would not be visible. Also, we have not plotted $\uparrow \downarrow \boldsymbol{\epsilon}_{-}$ as it coincides with $\downarrow \uparrow \boldsymbol{\epsilon}_{+}$. In all these cases, the two approaches also agree.

particular choice of the 4-vector potential and carry out the corresponding numerical calculation using the semiclassical method and the Volkov-states method. We set $a_{1}^{\mu}=\left\{0, a_{x}, 0,0\right\}, a_{2}^{\mu}=\left\{0,0, a_{y}, 0\right\}, k^{\mu}=\left\{\omega_{0}, 0,0,-\omega_{0}\right\}$, and

$$
\begin{gathered}
f_{1}(\varphi)=d(\varphi) \cos (\varphi), \\
f_{2}(\varphi)=d(\varphi) \sin (\varphi), \\
d(\varphi)= \begin{cases}\sin ^{4}\left(\frac{\varphi}{2 N}\right) & 0<\varphi<2 \pi N \\
0 & \text { otherwise. }\end{cases}
\end{gathered}
$$

That is, we choose a pulse with envelope $d(\varphi)$ and negative helicity (right-handed) circular polarization [38]. We define the polarizations of the outgoing light as

$$
\boldsymbol{\epsilon}_{ \pm}=\frac{1}{\sqrt{2}}\left(\boldsymbol{\epsilon}_{1} \pm i \boldsymbol{\epsilon}_{2}\right)
$$

where

$$
\epsilon_{1}=\frac{\hat{\boldsymbol{y}} \times \boldsymbol{k}}{|\hat{\boldsymbol{y}} \times \boldsymbol{k}|},
$$

with $\hat{y}$ being the unit vector in the $y$ direction, and

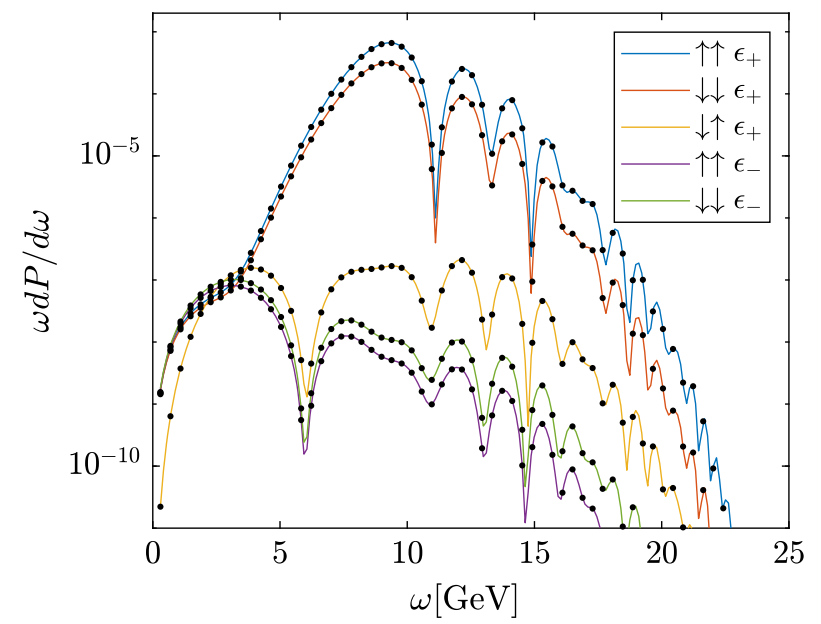

FIG. 2. Integrated intensity of radiation as in Fig. 1 but with a collimation angle of $0.1 / \gamma$, as explained in the text.

$$
\boldsymbol{\epsilon}_{2}=\frac{\boldsymbol{k} \times \boldsymbol{\epsilon}_{1}}{\left|\boldsymbol{k} \times \boldsymbol{\epsilon}_{1}\right|} .
$$

According to this choice, $\boldsymbol{\epsilon}_{1}$ and $\boldsymbol{\epsilon}_{2}$ are unit vectors orthogonal to each other and to $\boldsymbol{k}$ such that, if $\boldsymbol{k}$ lies along the $z$ axis, they indicate the polarization along the $x$ and $y$ directions, respectively. The $\boldsymbol{\epsilon}_{ \pm}$basis corresponds to circular polarization with helicity of \pm 1 . As the spin basis we have chosen a quantization axis along the $z$ direction such that $\phi$ may be chosen as $\left(\begin{array}{ll}1 & 0\end{array}\right)^{T}$ or $\left(\begin{array}{ll}0 & 1\end{array}\right)^{T}$, denoted by $\uparrow$ and $\downarrow$, respectively, in the figures. We set $a_{x}=a_{y}=m \xi / e$, where $\xi$ is the classical nonlinearity parameter, which we have set as $\xi=1, N=5$, and the electron energy $\varepsilon=30 \mathrm{GeV}$ for Figs. 1-4. Since the typical emission angles are small, we write $k_{x}=\omega \theta_{x}$ and $k_{y}=\omega \theta_{y}$ and then $d \Omega=d \theta_{x} d \theta_{y}$. In Fig. 1 we have restricted the angular integration such that $\left|\theta_{x}\right|<(\xi+3) / \gamma$, where $\gamma$ is the initial Lorentz factor of the electron, and the same for $\theta_{y}$ such that nearly all emitted radiation is included. In this figure we compare the semiclassical approach based on the formulas of Baier, Katkov and Strakhovenko, with the results obtained using the Volkov states. The results indicate nearly perfect agreement between the two approaches, which is expected since the motion in a plane wave is intrinsically semiclassical [12]. In Fig. 2 we do the same but restrict the emission angles over a smaller interval (collimation) i.e., $\left|\theta_{x}\right|<0.1 / \gamma$ and the same for $\theta_{y}$. In this case the emitted radiation with negative helicity is highly suppressed, and therefore we plot the results on a logarithmic scale. This is expected due to angular momentum conservation along the $z$ axis. Since the electron flipping its spin is unlikely for ultrarelativistic electrons [37], the outgoing light must have opposite helicity as that of the laser field to conserve angular momentum. Finally, the agreement between the semiclassical method and the Volkov-state method in this case indicates an agreement of the two approaches also at the 


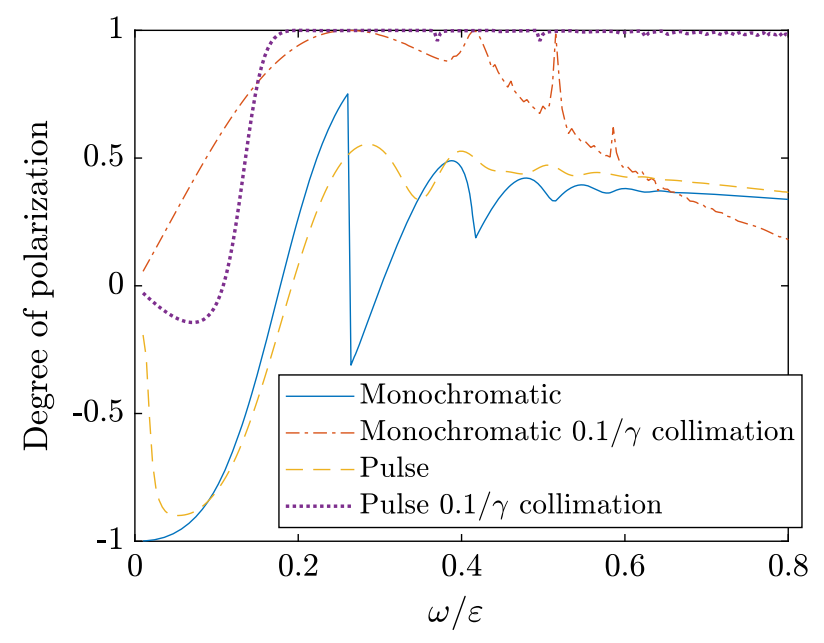

FIG. 3. Degree of circular polarization of the Compton scattered radiation for nonlinear Compton scattering in a monochromatic wave with and without angular collimation, and in the short pulse described in the text with and without angular collimation.

level of angularly resolved spectra. In Fig. 3 we show how the collimation affects the degree of circular polarization, defined as

$$
\mathcal{P}=\frac{\frac{d P^{+}}{d \omega}-\frac{d P^{-}}{d \omega}}{\frac{d P^{+}}{d \omega}+\frac{d P^{-}}{d \omega}} .
$$

We compare with the result found in [39], obtained in the case of the monochromatic wave, and see that in the short pulse one reaches a slightly smaller value of the degree of polarization around the first harmonic. By checking the results for the monochromatic case with a finite number of cycles, we also find agreement with the result of [39], and in addition, it is seen that the behavior close to $\omega=0$ is due to the finite opening angle of $\left|\theta_{x}\right|<(\xi+3) / \gamma$ mentioned above. That is, radiation below the value of $\omega$ where the curve changes drastically is emitted outside the chosen angular region. Since the analytical results with a finite opening angle are not presented in [39], we calculate this numerically using our approach with a finite number of cycles, with a number great enough to ensure that the result has converged. In this case, the spectrum of radiation consists of narrow peaks around the harmonic peaks, the position of which can also be seen from the curve labeled "Monochromatic." The harmonic peaks are located in the positions where the degree of polarization changes abruptly. The peaks seen in the degree of polarization in the monochromatic case with collimation coincide with the position of the harmonic peaks; i.e., most of the emitted radiation occurs when the degree of polarization is also large. Therefore, in both the cases of the pulse and the monochromatic wave, an effective degree of polarization close to unity is achieved. In Fig. 4 we show how the degree of

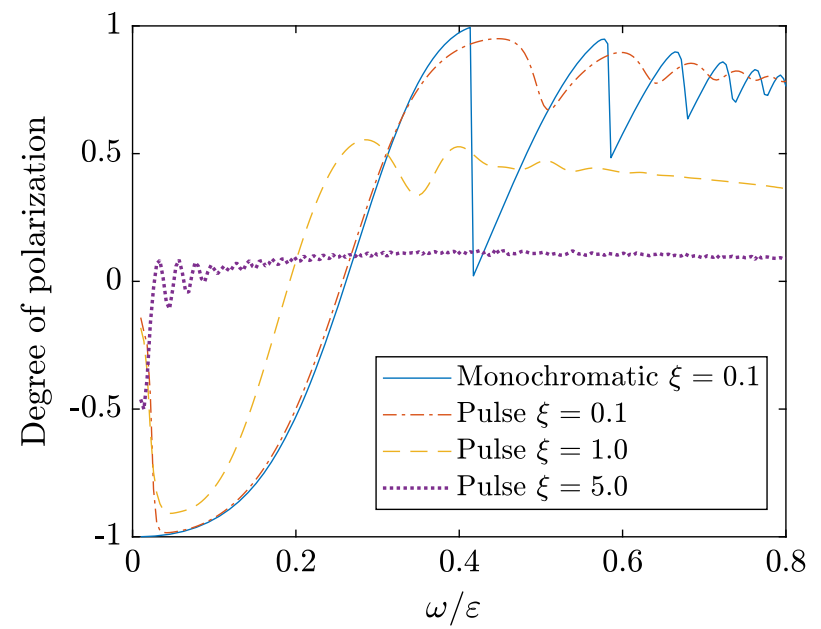

FIG. 4. Degree of circular polarization of the Compton scattered radiation for nonlinear Compton scattering in a monochromatic wave without collimation, for different values of the $\xi$ parameter in the pulse.

polarization decreases when the parameter $\xi$ is increased. Note also that in the monochromatic case, when $\xi$ is small, one achieves fully polarized light around the first harmonic. The method presented here is particularly useful as we require that $\xi$ is of the order of 1 in such a way that the emission of high harmonics is suppressed. Moreover, at $\xi \sim 1$ the total probability of emission is of the order of $2 \pi \alpha N$ [6] such that the obtained results are valid even for relatively long pulses as long as multiple photon emission is negligible. At the same time, this also implies that in the situations discussed above, one cannot use the often used local constant field approximation, as this requires at least that $\xi \gg 1$ (see e.g., [40,41]), and the semiclassical method presented here is a simple method to obtain accurate values of the degree of polarization which is valid also for external fields of complex spacetime structure (see also Refs. [42-44] for an alternative applicable method).

\section{POLARIZATION IN A BENT CRYSTAL}

Bent crystals can be used to steer an electron or positron beam along a circular arc as investigated in e.g., [45-48]. Also, the possibility of polarizing an electron/positron beam as in a storage ring through synchrotron radiation was discussed in e.g., [49]. The polarization of the beam is a quantum effect and depends on the parameter $\chi=e \sqrt{\left|\left(F^{\mu \nu} p_{\nu}\right)^{2}\right|} / m^{3}$, where $F^{\mu \nu}$ is the electromagnetic field tensor of the background field and $p^{\mu}$ the electron four momentum. The size of this parameter signifies the importance of quantum effects in the emission process such as spin effects and photon recoil. When this parameter is on the order of unity, polarization effects will become important even after a few emissions. In a conventional synchrotron this parameter is small, and therefore polarization of the beam happens through many emissions with 


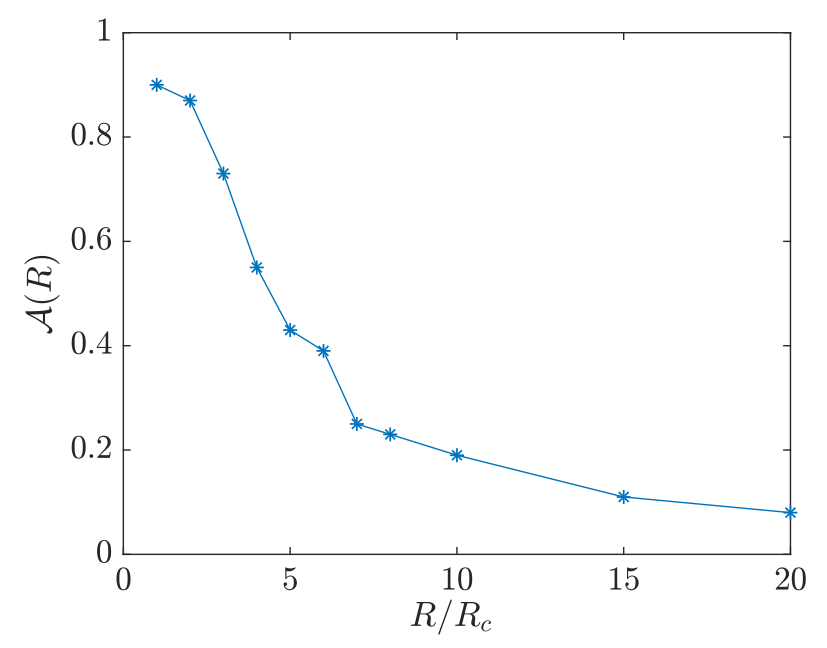

FIG. 5. The maximum possible transverse polarization that can be obtained for a positron with the initial conditions mentioned in the text, depending on the bending radius $R$ of the crystal in units of the Tsyganov critical radius $R_{c}$.

subsequent reaccelerations using rf cavities. In [49] it was assumed that the crystal was bent close to the so-called Tsyganov critical radius which we will define as

$$
R_{c}=\frac{\varepsilon d_{p}}{2 U_{0}}
$$

where $d_{p}$ is the distance between two symmetry planes in the crystal and $U_{0}$ is the corresponding potential energy depth. This radius corresponds to the radius at which the strength of the force from the electric field between the planes, estimated as $2 U_{0} / d_{p}$, can no longer provide the necessary centripetal force to sustain the circular motion. Below we consider the motion of a positron between two (110) planes in germanium such that $d_{p}=2.0 \AA$ and $U_{0}=35.73 \mathrm{eV}$. According to the above discussion, the Tsyganov critical radius is roughly the smallest bending radius at which channeling is still possible in the crystal. In this case the radiation and polarization characteristics are that of the constant magnetic field which produces the same bending radius of the trajectory, and therefore the largest possible polarization is given by $8 /(5 \sqrt{3})$ [29], when $\chi \ll 1$ [50]. Conversely, when the bending radius becomes large, one must recover the case of the flat crystal, which does not produce any beam polarization. With the presented approach we demonstrate that one can predict the polarization properties for any bending radius $R$, and not only for the extreme case close to the critical radius. In an experiment the average polarization will depend on the angular distribution of particles when entering the crystal. Thus, we will only apply the approach in the case of a single particle starting with an angle of 0 and a distance of $u_{0}=0.083 \AA$ from the plane (this value corresponds to the thermal vibrational amplitude of the nuclei in the crystal lattice). The maximum polarization that can be asymptotically obtained, $\mathcal{A}$, is given by $[28,49]$

$$
\mathcal{A}=\frac{W_{\uparrow \downarrow}-W_{\downarrow \uparrow}}{W_{\uparrow \downarrow}+W_{\downarrow \uparrow}},
$$

where $W_{f i}$ denotes the total transition rate from state $i$ to state $f$. The quantity $W_{f i}$ for different initial and final spin quantum numbers can be found from Eq. (13) by integrating over angles and photon energies, and by summing over the photon polarization, using a finite piece of trajectory. This formula comes about if it is assumed that the positron has its energy replenished between each radiation emission, as is the case in a synchrotron. With crystals, this would require several thin crystals with accelerating structures in between. We integrated the Lorentz force equation of motion using the electric field obtained from the continuum potential $[6,51]$, such that the electric field in the unbent crystal is along the $x$ direction. We then offset the plane along a circular arc in the $x z$ plane, which at the leading order in the small quantity $L / R$, where $L$ is the crystal length, means that the bending follows the curve $x=z^{2} / 2 R$. One may use this approximation as the total deflection angle $L / R$ is small in a realistic scenario. Due to symmetry, the electric field points along the radius of bending, and using Gauss' law one can show that as long as the distance to the plane is much smaller than the bending radius $R$, the electric field component along the radius of bending is the same as the electric field in the unbent case evaluated at the same distance from the plane. The nonzero components of the electric field are then

$$
\begin{gathered}
E_{x}(x, y, z)=E_{\text {cont }}\left(x-\frac{z^{2}}{2 R}\right), \\
E_{z}(x, y, z)=-\frac{z}{R} E_{\text {cont }}\left(x-\frac{z^{2}}{2 R}\right) .
\end{gathered}
$$

Here, $E_{\text {cont }}\left(x-z^{2} / 2 R\right)$ is the electric field obtained from the continuum potential, in the Doyle-Turner approximation [52-54], which depends only on the coordinate transverse to the planes (the $x$ coordinate in the considered case). We used a piece of trajectory with roughly 10 periods of oscillation, which was adequate for convergence of the integrals. Moreover, we have integrated over an angular region such that $\boldsymbol{v}_{\perp}(t)$ is contained in the region, with an additional angle of $10 / \gamma$ in each direction. This turned out numerically to be sufficient to cover all of the emitted radiation. In Fig. 5 we show the result for a $50 \mathrm{GeV}$ positron with the mentioned initial conditions. It should be mentioned that this maximum polarization is only achievable under the same circumstances as in a storage ring, i.e., a short piece of crystal where radiation occurs, and subsequently a replenishment of the lost energy so that the particles have the nominal energy before entering a crystal again. It is seen that for a strong bending of the 
crystal, one approaches the value in the constant field of $8 /(5 \sqrt{3})$. While we show only the example of a single trajectory, the method would allow us to study radiation reaction in a bent crystal where the effects of polarization of the beam would be essential. We refer the reader to Refs. [3,4] for recent experimental studies of radiation reaction in straight crystals.

\section{CONCLUSION}

In conclusion we have presented a method to rewrite the semiclassical formulas of Baier, Katkov and Strakhovenko, which facilities their numerical implementation for arbitrary discrete quantum numbers of the particles. This then allows for the calculation of radiation emission with arbitrary initial and final electron spins, and with arbitrary polarization of the emitted photon when knowing only the classical trajectory of the electron in the background field. In this way, one does not have to know the Dirac wave function in the background field, which is typically an impossible task for realistic field configurations.

First, we have compared the obtained formulas for a case where an exact solution of the Dirac equation is known, namely the plane-wave field, and find nearly perfect agreement between the two methods, corroborating the idea that the motion in a plane wave is intrinsically quasiclassical. As an example, we considered the case of the transfer of circular polarization of the radiation, when an electron beam head-on scatters on a short circularly polarized pulse, with the conclusion that the shortness of the pulse implies a slightly lower degree of polarization as compared to the monochromatic-field case. However, much higher degrees of polarization are observed for the photons emitted approximately along the initial direction of propagation of the electrons, in agreement with angular momentum conservation. Finally, we considered the case of a bent crystal and showed how one can calculate the degree of polarization of the positron beam for an arbitrary bending radius of the crystal.

\section{ACKNOWLEDGMENTS}

T. N. W. was supported by the Alexander von HumboldtStiftung. In addition, T. N. W. acknowledges the support of NVIDIA Corporation with the donation of the Titan V GPU used for this research.
[1] J. M. Cole et al., Experimental Evidence of Radiation Reaction in the Collision of a High-Intensity Laser Pulse with a Laser-Wakefield Accelerated Electron Beam, Phys. Rev. X 8, 011020 (2018).

[2] K. Poder et al., Experimental Signatures of the Quantum Nature of Radiation Reaction in the Field of an Ultraintense Laser, Phys. Rev. X 8, 031004 (2018).

[3] T. N. Wistisen, A. Di Piazza, H. V. Knudsen, and U. I. Uggerhøj, Experimental evidence of quantum radiation reaction in aligned crystals, Nat. Commun. 9, 795 (2018).

[4] T. N. Wistisen, A. Di Piazza, C. F. Nielsen, A. H. Sørensen, and U. I. Uggerhøj (CERN NA63 Collaboration), Quantum radiation reaction in aligned crystals beyond the local constant field approximation, Phys. Rev. Res. 1, 033014 (2019).

[5] H. Abramowicz et al., Letter of intent for the LUXE experiment, arXiv:1909.00860.

[6] V. N. Baier, V. M. Katkov, and V. M. Strakhovenko, Electromagnetic Processes at High Energies in Oriented Single Crystals (World Scientific, Singapore, 1998).

[7] A. Belkacem, N. Cue, and J. C. Kimball, Theory of crystalassisted radiation and pair creation for imperfect alignment, Phys. Lett. A 111, 86 (1985).

[8] T. N. Wistisen, Interference effect in nonlinear Compton scattering, Phys. Rev. D 90, 125008 (2014).

[9] D. M. Volkov, On a class of solutions of the dirac equation, Z. Phys. 94, 250 (1935).

[10] M. Boca and V. Florescu, The completeness of Volkov spinors, Rom. J. Phys. 55, 511 (2010).
[11] A. Di Piazza, Completeness and orthonormality of the Volkov states and the Volkov propagator in configuration space, Phys. Rev. D 97, 056028 (2018).

[12] V. I. Ritus, Quantum effects of the interaction of elementary particles with an intense electromagnetic field, J. Sov. Laser Res. 6, 497 (1985).

[13] D. Seipt and B. Kämpfer, Two-photon Compton process in pulsed intense laser fields, Phys. Rev. D 85, 101701 (2012).

[14] F. Mackenroth and A. Di Piazza, Nonlinear Compton scattering in ultrashort laser pulses, Phys. Rev. A 83, 032106 (2011).

[15] V. Dinu and G. Torgrimsson, Single and double nonlinear Compton scattering, Phys. Rev. D 99, 096018 (2019).

[16] M. Boca and V. Florescu, Nonlinear Compton scattering with a laser pulse, Phys. Rev. A 80, 053403 (2009).

[17] D. Seipt and B. Kämpfer, Nonlinear Compton scattering of ultrashort intense laser pulses, Phys. Rev. A 83, 022101 (2011).

[18] A. Di Piazza, C. Müller, K. Z. Hatsagortsyan, and C. H. Keitel, Extremely high-intensity laser interactions with fundamental quantum systems, Rev. Mod. Phys. 84, 1177 (2012).

[19] C. Harvey, T. Heinzl, and A. Ilderton, Signatures of highintensity Compton scattering, Phys. Rev. A 79, 063407 (2009).

[20] H. Hu, C. Müller, and C. H. Keitel, Complete QED Theory of Multiphoton Trident Pair Production in Strong Laser Fields, Phys. Rev. Lett. 105, 080401 (2010). 
[21] S. Meuren, C. H. Keitel, and A. Di Piazza, Semiclassical picture for electron-positron photoproduction in strong laser fields, Phys. Rev. D 93, 085028 (2016).

[22] A. Ilderton, Trident Pair Production in Strong Laser Pulses, Phys. Rev. Lett. 106, 020404 (2011).

[23] A. I. Voroshilo, S. P. Roshchupkin, and V. N. Nedoreshta, Parametric interference Compton effect in two pulsed laser waves, J. Phys. B 48, 055401 (2015).

[24] K. Krajewska and J.Z. Kamiński, Compton process in intense short laser pulses, Phys. Rev. A 85, 062102 (2012).

[25] K. Krajewska and J. Z. Kamiński, Breit-Wheeler process in intense short laser pulses, Phys. Rev. A 86, 052104 (2012).

[26] D. Seipt, D. Del Sorbo, C. P. Ridgers, and A. G. R. Thomas, Theory of radiative electron polarization in strong laser fields, Phys. Rev. A 98, 023417 (2018).

[27] A. Angioi, F. Mackenroth, and A. Di Piazza, Nonlinear single Compton scattering of an electron wave packet, Phys. Rev. A 93, 052102 (2016).

[28] J. D. Jackson, On understanding spin-flip synchrotron radiation and the transverse polarization of electrons in storage rings, Rev. Mod. Phys. 48, 417 (1976).

[29] A. A. Sokolov and I. M. Ternov, Sinkhrotronnoie izluchenie, Akad. Nauk SSSR, Moskovskoie Obshchestvo Ispytatelei prirody. Sektsia Fiziki. Sinkhrotron Radiat 228, 1 (1966) [Synchrotron radiation], https://ui.adsabs.harvard .edu/abs/1966siiz.book.....S/abstract.

[30] D. Del Sorbo, D. Seipt, T. G. Blackburn, A. G. R. Thomas, C. D. Murphy, J. G. Kirk, and C. P. Ridgers, Spin polarization of electrons by ultraintense lasers, Phys. Rev. A 96, 043407 (2017).

[31] Y. F. Li, R. Shaisultanov, K. Z. Hatsagortsyan, F. Wan, C. H. Keitel, and J. X. Li, Ultrarelativistic Electron-Beam Polarization in Single-Shot Interaction with an Ultraintense Laser Pulse, Phys. Rev. Lett. 122, 154801 (2019).

[32] A. F. Elishev et al., Steering of charged particle trajectories by a bent crystal, Phys. Lett. B 88, 387 (1979).

[33] E. N. Tsyganov, Estimates of Cooling and Bending Processes for Charged Particle Penetration through a Mono Crystal (Fermilab TM-684, Batavia, 1976).

[34] J. D. Jackson, Classical Electrodynamics, 3rd ed. (John Wiley \& Sons, New Jersey, 1991).

[35] T. N. Wistisen and A. Di Piazza, Impact of the quantized transverse motion on radiation emission in a Dirac harmonic oscillator, Phys. Rev. A 98, 022131 (2018).

[36] T. N. Wistisen and A. Di Piazza, Complete treatment of single-photon emission in planar channeling, Phys. Rev. D 99, 116010 (2019).

[37] V. B. Berestetskii, E. M. Lifshitz, and L. P. Pitaevskii, Relativistic Quantum Theory (Elsevier, New York, 1971).

[38] Due to the presence of the finite pulse shape $d(\varphi)$ the electric field of the wave is not, rigorously speaking, circularly polarized. For the sake of simplicity, however, in the numerical examples we choose sufficiently long pulses that we can ignore this subtlety.

[39] D. Yu. Ivanov, G. L. Kotkin, and V. G. Serbo, Complete description of polarization effects in emission of a photon by an electron in the field of a strong laser wave, Eur. Phys. J. C 36, 127 (2004).
[40] A. Di Piazza, M. Tamburini, S. Meuren, and C. H. Keitel, Implementing nonlinear Compton scattering beyond the local-constant-field approximation, Phys. Rev. A 98, 012134 (2018).

[41] A. Ilderton, B. King, and D. Seipt, Extended locally constant field approximation for nonlinear Compton scattering, Phys. Rev. A 99, 042121 (2019).

[42] A. Di Piazza, Ultrarelativistic Electron States in a General Background Electromagnetic Field, Phys. Rev. Lett. 113, 040402 (2014).

[43] A. Di Piazza, Analytical tools for investigating strong-field QED processes in tightly focused laser fields, Phys. Rev. A 91, 042118 (2015).

[44] A. Di Piazza, First-order strong-field QED processes in a tightly focused laser beam, Phys. Rev. A 95, 032121 (2017).

[45] U. Wienands, T. W. Markiewicz, J. Nelson, R. J. Noble, J. L. Turner, U. I. Uggerhøj, T. N. Wistisen, E. Bagli, L. Bandiera, G. Germogli, V. Guidi, A. Mazzolari, R. Holtzapple, and M. Miller, Observation of Deflection of a Beam of Multi-GeV Electrons by a Thin Crystal, Phys. Rev. Lett. 114, 074801 (2015).

[46] T. N. Wistisen, U. I. Uggerhøj, U. Wienands, T. W. Markiewicz, R. J. Noble, B. C. Benson, T. Smith, E. Bagli, L. Bandiera, G. Germogli, V. Guidi, A. Mazzolari, R. Holtzapple, and S. Tucker, Channeling, volume reflection, and volume capture study of electrons in a bent silicon crystal, Phys. Rev. Accel. Beams 19, 071001 (2016).

[47] T. N. Wistisen, R. E. Mikkelsen, U. I. Uggerhøj, U. Wienands, T. W. Markiewicz, S. Gessner, M. J. Hogan, R. J. Noble, R. Holtzapple, S. Tucker, V. Guidi, A. Mazzolari, E. Bagli, L. Bandiera, and A. Sytov (SLAC E-212 Collaboration), Observation of Quasichanneling Oscillations, Phys. Rev. Lett. 119, 024801 (2017).

[48] A. Mazzolari, E. Bagli, L. Bandiera, V. Guidi, H. Backe, W. Lauth, V. Tikhomirov, A. Berra, D. Lietti, M. Prest, E. Vallazza, and D. De Salvador, Steering of a Sub-GeV Electron Beam through Planar Channeling Enhanced by Rechanneling, Phys. Rev. Lett. 112, 135503 (2014).

[49] V. G. Baryshevskiǔ and V. V. Tikhomirov, Synchrotrontype radiation processes in crystals and polarization phenomena accompanying them, Sov. Phys. Usp. 32, 1013 (1989).

[50] V. N. Baier, Radiative polarization of electrons in storage rings, Sov. Phys. Usp. 14, 695 (1972).

[51] J. Lindhard, Influence of crystal lattice on motion of energetic charged particles, K. Dan. Vidensk. Selsk. Mat. Fys. Medd. 34, 1 (1965).

[52] P. A. Doyle and P.S. Turner, Relativistic Hartree-Fock X-ray and electron scattering factors, Acta Crystallogr. Sect. A 24, 390 (1968).

[53] A. L. Avakian, N. K. Zhevago, and S. Yan, Emission of electrons and positrons in the axial semichanneling, J. Exp. Theor. Phys. 82, 573 (1982).

[54] S. P. Møller, High-energy channeling-Applications in beam bending and extraction, Nucl. Instrum. Methods Phys. Res., Sect. A 361, 403 (1995). 